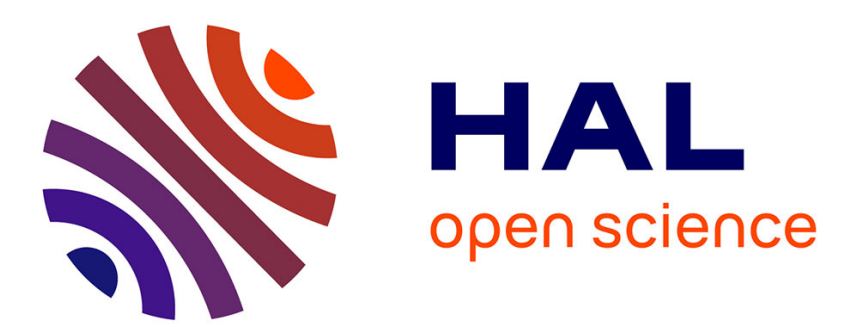

\title{
Direct adaptive control design for one-degree-of-freedom complementary-slackness jugglers
}

\author{
Arturo Zavala-Río, Bernard Brogliato
}

\section{To cite this version:}

Arturo Zavala-Río, Bernard Brogliato. Direct adaptive control design for one-degree-of-freedom complementary-slackness jugglers. Automatica, 2001, 37 (7), pp.1117-1123. 10.1016/S00051098(01)00061-9 . hal-02280051

\section{HAL Id: hal-02280051 \\ https://hal.science/hal-02280051}

Submitted on 5 Sep 2019

HAL is a multi-disciplinary open access archive for the deposit and dissemination of scientific research documents, whether they are published or not. The documents may come from teaching and research institutions in France or abroad, or from public or private research centers.
L'archive ouverte pluridisciplinaire HAL, est destinée au dépôt et à la diffusion de documents scientifiques de niveau recherche, publiés ou non, émanant des établissements d'enseignement et de recherche français ou étrangers, des laboratoires publics ou privés. 


\title{
Direct adaptive control design for one-degree-of-freedom complementary-slackness jugglers
}

\author{
Arturo Zavala-Rio ${ }^{\mathrm{a}, *}$, Bernard Brogliato ${ }^{\mathrm{b}}$ \\ ${ }^{a}$ Universidad Autónoma de San Luis Potosí, CIEP-FI, Av. Dr. Manuel Nava 8, Zona Universitaria, 78290 San Luis Potosí, S.L.P., Mexico \\ ${ }^{\mathrm{b}}$ Laboratoire d'Automatique de Grenoble (UMR 5528 CNRS-INPG), ENSIEG, BP 46, 38402 St. Martin d'Héres, France
}

\begin{abstract}
This note deals with the problem of controlling a simple one-degree-of-freedom (1-dof) juggling robot (a system that belongs to the class of nonsmooth hybrid complementary-slackness dynamical systems), when some physical parameters such as the object mass and the restitution coefficient are not exactly known. The proposed adaptive controller is based on so-called dead-beat viable controllers previously studied, in which the sequence of desired "robot" pre-impact velocities is suitably modified. The dynamics of a simple 1-dof hopper is shown to be controllable by the proposed control algorithm. Numerical simulations support the theoretical results.
\end{abstract}

Keywords: Complementary-slackness; Juggling; Non-smooth mechanics; Adaptive control

\section{Introduction}

The analysis and control of nonsmooth hybrid mechanical systems with unilateral constraints (that give rise to complementarity conditions) has recently been the object of various studies (ten Dam, Dwarshuis, \& Willems, 1997; van der Schaft \& Schumacher, 1996), and still represents an open topic with many problems unsolved yet. In this note we focus our attention on a very simple case of such complementary-slackness systems, a one-degree-of-freedom (1-dof) juggler. This belongs to a much more general class of nonsmooth models named complementary-slackness juggling mechanical systems (Brogliato \& Zavala-Río, 2000), that encompasses "true" jugglers as well as hoppers, nonprehensile manipulators, robots with passive dynamical environments, simple models of controlled buildings, etc. In particular it is shown that the 1-dof juggler dynamics matches with that of a (simplified) 1-dof hopper. The control problem for such systems has been studied in Zavala-Río and Brogliato (1999) and Zavala-Río (1997)

\footnotetext{
*Corresponding author. Tel./fax: + 52-4-817-3381
}

E-mail address: azavala@uaslp.mx (A. Zavala-Rio). where a class of hybrid dead-beat controllers has been proposed and thoroughly analyzed (see also these references for a review of juggler control). In Brogliato and Zavala-Río (2000) a more general point of view on complementary-slackness juggling systems has been taken, and some controllability criteria have been proposed. Roughly, they hinge on the analysis of an impact Poincare map that represents the object dynamics from one impact to the next, considering the pre-impact velocity of the robot as an intermediate input. When this discrete-time map is controllable with the robot velocity as input, the object has been defined to be controllable via impacts. In this note, it is shown that a suitable extension of the dead-beat controllers allows one to derive an adaptive scheme which permits to relax the a priori knowledge on the object mass and on the restitution coefficient, while guaranteeing convergence of the process state towards its desired (discrete-time) orbit.

\section{One-degree-of-freedom juggler}

Let us begin by recalling the 1-dof juggler dynamics (see Fig. 1)

$$
\begin{aligned}
& m_{1} \ddot{y}_{1}=-m_{1} g+\lambda, \\
& m_{2} \ddot{y}_{2}=-m_{2} g+u-\lambda,
\end{aligned}
$$




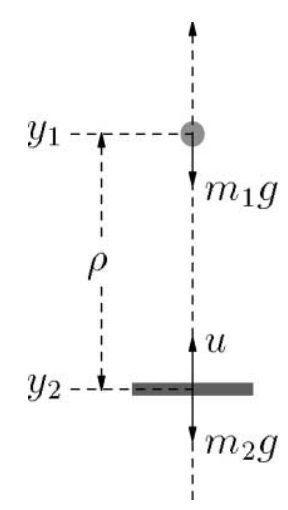

Fig. 1. One-dof juggler.

$\rho \triangleq y_{1}-y_{2} \geqslant 0, \quad \lambda \geqslant 0, \lambda \rho=0$,

$\dot{\rho}\left(t_{k}^{+}\right)=-e \dot{\rho}\left(t_{k}^{-}\right)$,

where the object and the robot dynamics are in (1) and (2) with $u$ as the control input force, the complementarity conditions between the distance $\rho$ and the Lagrange multiplier $\lambda$ are in (3), and the restitution law is in (4) where $e \in[0,1]$ is the restitution coefficient. The complementarity conditions in (3) mean that all gluing or magnetic effects are excluded from the model, so that the contact force $\lambda$ has to be zero whenever the distance $\rho$ becomes positive. On the contrary a positive contact force $\lambda$ implies contact between the two bodies. The name complementary-slackness systems is simply taken from some standard terminology in convex analysis (Rockafellar, 1970). Collisions take place at impact times $t_{k}$ if and only if $\rho\left(t_{k}\right)=0$ and $\dot{\rho}\left(t_{k}^{-}\right)<0$ (Brogliato, 1999), yielding (Zavala-Río, 1997; Brach, 1991)

$\dot{y}_{1}\left(t_{k}^{+}\right)=\frac{m-e}{1+m} \dot{y}_{1}\left(t_{k}^{-}\right)+\frac{1+e}{1+m} \dot{y}_{2}\left(t_{k}^{-}\right)$,

$\dot{y}_{2}\left(t_{k}^{+}\right)=\frac{m(1+e)}{1+m} \dot{y}_{1}\left(t_{k}^{-}\right)+\frac{1-e m}{1+m} \dot{y}_{2}\left(t_{k}^{-}\right)$,

where $m \triangleq m_{1} / m_{2}$. Subsequently in this study, $f(k)$ will stand for $f$ 's post-impact values $f\left(t_{k}^{+}\right)\left(f\left(t_{k}\right)\right.$ if $f$ denotes a position). Pre-impact values $f\left(t_{k}^{-}\right)$will be referred explicitly.

Remark 1. Let us apply Lemma 4 of Brogliato and Zavala-Río (2000) to the 1-dof juggler dynamics, i.e. consider the new coordinate system $\left(q_{1}, q_{2}\right)$ where $q_{1}=y_{1}$ and $q_{2}=\rho$. Then (1)-(4) is transformed into

$\ddot{q}_{1}=-g+\bar{m}_{1} \lambda$,

$\ddot{q}_{2}=-\frac{u}{m_{2}}+(1+m) \bar{m}_{1} \lambda$,

$q_{2} \geqslant 0, \quad \lambda \geqslant 0, \lambda \rho=0$,

$\dot{q}_{2}\left(t_{k}^{+}\right)=-e \dot{q}_{2}\left(t_{k}^{-}\right)$,

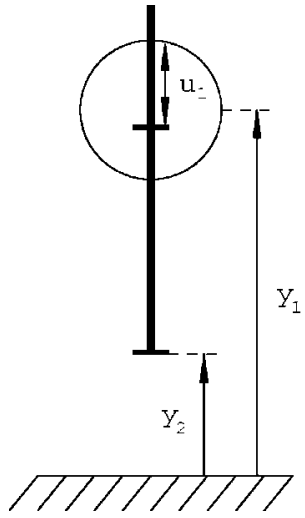

Fig. 2. One-dof hopper.

where $\bar{m}_{1}=1 / m_{1}$. Now, let us consider the 1 -dof hopper in Fig. 2. It is easy to show that in the coordinate system $\left(q_{1}, q_{2}\right)=\left(y_{c}, y_{2}\right)$, where $y_{c} \triangleq\left(m_{1} /\left(m_{1}+m_{2}\right)\right) y_{1}+\left(m_{2} /\right.$ $\left.\left(m_{1}+m_{2}\right)\right) y_{2}$, its dynamics are also modeled by (6) with $\bar{m}_{1}=1 /\left(m_{1}+m_{2}\right)$ and $u=m_{2} g+u_{1}$. Therefore, a dead-beat-algorithm-based input and impact control strategies equivalent to those to be presented in Sections 3 and 4 can be gotten for this simple 1-dof hopping robot. Let us notice that we have implicitly assumed that the bar is long enough so that $y_{1}(t)>0, \forall t \geqslant 0$, whatever the control may be. Clearly, further studies should incorporate $y_{1}-y_{2}>R$ for some $R>0$. But then one would have to deal with possible multiple shocks, which may complicate the wellposedness of the model (Brogliato, 1999). In Brogliato and Zavala-Río (2000), the multiple constraint case is discussed.

\section{Dead-beat force input}

The following proposition briefly recalls the control strategy proposed in Zavala-Río and Brogliato (1999) (see also other references therein). It mixes a finite-time convergent input based on the robot's controllability gramian inversion, and some logic to cope with the object's ballistic constraints. Such controllers may be seen as two-stages inputs (Brogliato \& Zavala-Río, 2000): the first stage is to design an intermediate control signal, that is the robot pre-impact velocity, whose desired value is denoted as $\dot{y}_{2}^{*}(k)$. The second stage is the design of the control input force $u$. In the next section, we shall focus on an adaptive version of $\dot{y}_{2}^{*}(k)$ that relaxes the a priori knowledge on certain physical parameters. Details on the meaning of the various terms appearing in the expression of the control input are given after Proposition 1. As pointed out in Brogliato and Zavala-Río (2000), other finite-time inputs can be designed. In the following, the signals with upper index $*$ denote values that the state $\left(y_{1}, y_{2}, \dot{y}_{1}, \dot{y}_{2}\right)$ is forced to track at all impact times. They differ from the signals with lower index $d$, which denote 
the desired trajectory of the object. The reason for this discrepancy is that one has to incorporate the ballistic constraints of the object in the control design (the least requirement being that the intersection between the desired discrete-time orbit and the real orbit of the object, is not empty).

Proposition 1. Consider the dynamical system in (1)-(4). Suppose that the initial conditions and $u$ are such that there exists an impact or contact time $t_{0} \geqslant 0$. Let $\left(y_{d}, \dot{y}_{d}\right)$ be the desired position and post-impact velocity of the object. Let us define the following control input:

$u=m_{2} g+m_{2} v$

$v=A_{k}\left(t-t_{k}\right)+B_{k}$

with

$A_{k}=\frac{6}{d_{k}^{2}}\left(\dot{y}_{2}^{*}(k+1)+\dot{y}_{2}(k)\right)-\frac{12}{d_{k}^{3}}\left(y^{*}(k+1)-y(k)\right)$,

$B_{k}=-\frac{2}{d_{k}}\left(\dot{y}_{2}^{*}(k+1)+2 \dot{y}_{2}(k)\right)$

$$
+\frac{6}{d_{k}^{2}}\left(y^{*}(k+1)-y(k)\right),
$$

$d_{k}=\frac{\dot{y}_{1}(k)+\varphi(k)}{g}$,

$\varphi(k)=\sqrt{\dot{y}_{1}^{2}(k)-2 g\left(y^{*}(k+1)-y(k)\right)}$,

$y^{*}(k+1)= \begin{cases}y_{d} & \text { if } h_{k}>y_{d}, \\ y(k)+r & \text { if } h_{k} \leqslant y_{d},\end{cases}$

$h_{k}= \begin{cases}y(k)+\frac{\dot{y}_{1}^{2}(k)}{2 g} & \text { if } \dot{y}_{1}(k)>0, \\ y(k) & \text { if } \dot{y}_{1}(k) \leqslant 0,\end{cases}$

$\forall k \geqslant 0$, where $y(k)=y_{1}(k)=y_{2}(k)($ since $\rho(k)=0)$, and $r$ and $\dot{y}_{2}^{*}(k+1)$ are chosen such that $y(k)+r<h_{k}$, and

$\dot{y}_{2}^{*}(k+1)>-\varphi(k)$,

$\forall k \geqslant 0$. Then,

(1) $\rho(t)=y_{1}(t)-y_{2}(t)>0, \forall t \in\left(t_{k}, t_{k}+d_{k}\right)$,

(2) $t_{k+1}=t_{k}+d_{k}$,

(3) $y(k+1)=y^{*}(k+1)$,

(4) $\dot{y}_{2}\left(t_{k+1}^{-}\right)=\dot{y}_{2}^{*}(k+1)$,

$\forall k \geqslant 0$. Moreover, if $\dot{y}_{2}^{*}(k+1)$ is defined as follows:

$\dot{y}_{2}^{*}(k+1)=\frac{1+m}{1+e} \dot{y}_{1}^{*}(k+1)+\frac{m-e}{1+e} \varphi(k)$, $\forall k \geqslant 0$, and

$\dot{y}_{1}^{*}(k+1)= \begin{cases}\dot{y}_{d} & \text { if } h_{k}>y_{d}, \\ \sqrt{\dot{y}_{d}^{2}+2 g\left(y_{d}-y(k)-r\right)} & \text { if } h_{k} \leqslant y_{d},\end{cases}$

$\forall k \geqslant 0$, with $\dot{y}_{d}>0$, then,

(5) $\dot{y}_{1}(k+1)=\dot{y}_{1}^{*}(k+1), \forall k \geqslant 0$,

(6) $\left(y(k+1), \dot{y}_{1}(k+1)\right)=\left(y_{d}, \dot{y}_{d}\right), \forall k \geqslant 1$.

Proof. See Zavala-Río and Brogliato (1999).

Let us provide some insights on properties (1)-(6) of Proposition 1:

- Property 1 means that no unexpected collision takes place at any time within $\left(t_{k}, t_{k}+d_{k}\right)$. This is known as the viability condition, and is very important for the overall scheme to work. In Zavala-Río (1997) and Wang (1993), more detailed and general explanations about viability and/or viability conditions can be found. These are fundamental in the study of vibro-impact systems (Babitsky, 1998) and have sometimes been forgotten (Shaw \& Rand, 1989; Masri \& Caughey, 1966).

- Property 2 means that, given a collision time $t_{k}$, the next impact takes place immediately after a flight time $d_{k}$. In other words, the flight times are predefined at every shock through the value of $d_{k}$ determined by (11). This expression is such that the object never goes upwards at impact times. The term $\varphi(k)$ in $(12)$ is a calculation (according to the ballistic trajectory of the object), in absolute value, of the object pre-impact velocity at $t_{k+1}$, i.e. $\varphi(k)=\left|\dot{y}_{1}\left(t_{k+1}^{-}\right)\right|$. It is calculated through $y^{*}(k+1)$ which in turn is chosen such that the object never be motionless at collision times (see (13) and (14)), hence $\varphi(k)>0, \forall k \geqslant 0$ (the object always goes downwards at controlled shock times).

- Property 3 means that the next impact position is arbitrarily predefined within the ballistic trajectory of the object through a suitable value of $y^{*}(k+1)$. The highest position that the object can reach during any flight time is given by $h_{k}$ (see (14)). It would not make any sense to try to hit the object at a position that is not on its trajectory (a position higher than $h_{k}$ ). Eq. (13) assures that $y^{*}(k+1)<h_{k}$.

- Property 4 means that the surface is forced to collide the object with an arbitrary pre-impact velocity through the value of $\dot{y}_{2}^{*}(k+1)$ satisfying (15). Such condition essentially arises from the pre-impact velocity necessary condition: $\dot{\rho}\left(t_{k+1}^{-}\right)<0$. Furthermore, it appears to be fundamental in the proofs of viability and contact loss too (Zavala-Río \& Brogliato, 1999). 
- Property 5 means that the object post-impact velocities are arbitrarily predefined through the value of $\dot{y}_{1}^{*}(k+1)$ in (16) (provided that (15) is satisfied, which is ensured by (17)).

- Property 6 means that the object impact Poincaré map orbit converges to the desired trajectory $\left(y_{d}, \dot{y}_{d}\right)$ after one impact.

\section{Direct adaptive control}

In this section, the signal $\dot{y}_{2}^{*}(\cdot)$ that appears in (9) and (10) is suitably modified to relax its dependence on the restitution coefficient $e$ (see (16)).

Proposition 2. Let us define $L(k) \triangleq b+(1+M) v(k)+$ $(M-b) \varphi(k) / \dot{y}_{d}$, where $v(k) \triangleq \sqrt{2 g\left(y_{d}-y^{*}(k+1)\right)}$, and $M$ is any value larger than $m \triangleq m_{1} / m_{2}$. Let $\dot{y}_{2}^{*}(k+1)$ be defined as follows (instead of (16)):

$\dot{y}_{2}^{*}(k+1)=\dot{y}_{d} a(k)+b\left(\varphi(k)-\dot{y}_{d}\right)$,

where $\dot{y}_{d}>0$ and $b \in(-1,-0.5)$. $a(k)$ is an auxiliary state which dynamics are defined as

$a(k+1)=a(k)+\frac{c}{\dot{y}_{d}}\left(\varphi(k)-\dot{y}_{d}\right)$

with $c \in(b+0.5,0)$. Its initial conditions must be defined according to the following criterion:

(1) If $h_{0}>y_{d}: a(0)$ is taken such that $a(0) \geqslant L(0)$ (and (19) is computed at every impact $\forall k \geqslant 0$ ).

(2) If $h_{0} \leqslant y_{d}$ : first, at $k=0, a(0)$ is taken such that $a(0) \geqslant L(0)$ and (19) is not computed; next, at $k=1$, $a(1)$ is taken such that $a(1) \geqslant L(1)$ (and (19) is computed at every impact $\forall k \geqslant 1$ ).

Then, for any $e \in[0,1]$ and $m \in[0, M): y(k)=y_{d}, \forall k \geqslant 2$, and $\lim _{k \rightarrow \infty}\left(\dot{y}_{1}(k), a(k)\right)=\left(\dot{y}_{d}, a^{*}\right)$, where $a^{*}=(1-e+$ $2 m) /(1+e)$.

Before the proof of Proposition 2, let us comment on the meaning of $L(k)$. The expression defined as $L(k)$ is such that $a(k) \geqslant L(k)$ represents a sufficient condition (given the uncertainty of $e$ and $m$ ) to assure $h_{k+1}>y_{d}$. This will be made clear within the proof. The importance of ensuring $h_{k}>y_{d}$ during two initial subsequent impacts will also be highlighted within the proof.

Proof. From (5a), the object dynamics integrated in time from $t_{k}$ to $t_{k}+d_{k},(11)$, and properties 2 and 4 of Proposition 1 , we get

$\dot{y}_{1}(k+1)=-\frac{m-e}{1+m} \varphi(k)+\frac{1+e}{1+m} \dot{y}_{2}^{*}(k+1)$

which is a valid expression for any $y^{*}(k+1)<h_{k}$ and $\dot{y}_{2}^{*}(k+1)$ satisfying condition (15). Both conditions will appear to be satisfied later in the proof. Indeed, on the one hand, one can easily verify that the desired impact position trajectory defined in (13) yields, at every impact, values of $y^{*}(k+1)$ lower than $h_{k}$ (see (14)). On the other hand, the proposed expression for $\dot{y}_{2}^{*}(k+1)$ in (18) will be proved to guarantee: $\dot{y}_{1}(k+1)>0, \forall k \geqslant 0$. Then, from (20), it follows that:

$\dot{y}_{1}(k+1)>0 \Leftrightarrow \dot{y}_{2}^{*}(k+1)>\frac{m-e}{1+e} \varphi(k)$.

Notice that for any $m \geqslant 0$ and $e \in[0,1]:[(m-e) /(1+e)]$ $\varphi(k)>-\varphi(k)$. Hence, any value of $\dot{y}_{2}^{*}(k+1)$ greater than $[(m-e) /(1+e)] \varphi(k)$ is even greater than $-\varphi(k)$. From this fact and (21), we have

$$
\begin{aligned}
\dot{y}_{1}(k+1)>0 & \Leftrightarrow \dot{y}_{2}^{*}(k+1)>\frac{m-e}{1+e} \varphi(k) \\
& \Rightarrow \dot{y}_{2}^{*}(k+1)>-\varphi(k),
\end{aligned}
$$

which proves that any value or expression of $\dot{y}_{2}^{*}(k+1)$ guaranteeing $\dot{y}_{1}(k+1)>0$ satisfies condition (15) of Proposition 1. Now, substituting (18) into (20), we get

$\dot{y}_{1}(k+1)=\sigma \dot{y}_{d} a(k)+\alpha \varphi(k)-\sigma b \dot{y}_{d}$,

where: $\sigma \triangleq(1+e) /(1+m)$ and $\alpha \triangleq \sigma(b+1)-1$. The rest of the proof is divided in two cases depending on whether the initial conditions are such that $h_{0}>y_{d}$, or such that $h_{0} \leqslant y_{d}$ :

(1) Initial conditions such that $h_{0}>y_{d}$ : Let us for the moment suppose that $h_{k}>y_{d}, \forall k \geqslant 1$. Then, from property 3 of Proposition 1, (13) and (12), we have $y(k)=y^{*}(k)=y_{d}$ and $\varphi(k)=\dot{y}_{1}(k)>0, \forall k \geqslant 1$. Hence, Eq. (22) can be expressed as

$\varphi(k+1)=\sigma \dot{y}_{d} a(k)+\alpha \varphi(k)-\sigma b \dot{y}_{d}$,

$\forall k \geqslant 0$. Let us now define the error states $\varepsilon_{1}(k) \triangleq \varphi(k)$ $-\dot{y}_{d}$ and $\varepsilon_{2}(k) \triangleq a(k)-a^{*}$, and the error state vector $\varepsilon(k) \triangleq\left(\varepsilon_{1}(k), \varepsilon_{2}(k)\right)^{\mathrm{T}}$. Then, (23) and (19) can be expressed in the error state space as

$\varepsilon(k+1)=A \varepsilon(k)$,

where

$A=\left[\begin{array}{ll}\alpha & \sigma \dot{y}_{d} \\ \frac{c}{\dot{y}_{d}} & 1\end{array}\right]$.

The origin of (24) is an asymptotically stable equilibrium point if the characteristic polynomial of $A$, i.e. $P(z)=$ $|z I-A|=z^{2}+p_{1} z+p_{0}$, is Schur stable (all its roots have magnitude less than unity). From (25): $p_{1}=-1-\alpha$ and $p_{0}=\alpha-c \sigma$. Let us define $\hat{P}(s) \triangleq$ $(s-1)^{2} P((s+1) /(s-1))=\hat{p}_{2} s^{2}+\hat{p}_{1} s+\hat{p}_{0}$. We get: $\hat{p}_{2}=-c \sigma, \quad \hat{p}_{1}=2(1-\alpha+c \sigma)=2(2+\sigma(c-b-1))$, 
and $\hat{p}_{0}=2+2 \alpha-c \sigma=\sigma(2+2 b-c)$. It is well-known that if $\hat{P}(s)$ preserves the same degree as $P(z)$, i.e. $\hat{p}_{2} \neq 0$, then $P(z)$ is Schur stable if and only if $\hat{P}(s)$ is Hurwitz stable (all its roots have negative real part) (Bhattacharyya, Chapellat, \& Keel, 1995). One can easily verify that for any $m \in[0, M), e \in[0,1], b \in(-1,-0.5)$, and $c \in(b+0.5,0)$, we have: $\hat{p}_{i}>0, \forall i=0,1,2$, which is a sufficient condition for $\hat{P}(s)$ to be a second degree Hurwitz stable polynomial. Therefore, the proposed tuning criterion, i.e. $b \in(-1,-0.5)$ and $c \in(b+0.5,0)$, ensures the Schur stability of $P(z)$, guaranteeing the asymptotic stability of (24), hence $\lim _{k \rightarrow \infty} \varepsilon(k)=0$. To complete the proof (before the second initial condition case is treated), we still need to show that $h_{k}>y_{d}, \forall k \geqslant 1$ (recall that this was initially supposed) which is what validates (23) and (24), and gives rise to: $y(k)=y^{*}(k)=y_{d}, \forall k \geqslant 1$. First of all, notice that: $h_{k}>y_{d} \Rightarrow y^{*}(k+1)=y_{d} \quad$ (see (13)) $\Rightarrow y(k+1)=y_{d}$ (recall property 3 of Proposition 1$)$. Moreover: $\quad y(k+1)=y_{d} \quad$ and $\quad \dot{y}_{1}(k+1)>0$ imply $h_{k+1}>y_{d}$ (see (14)). Hence, all we need is to show that the proposed control input ensures $\dot{y}_{1}(k+1)>0, \forall k \geqslant 0$ (recall that $h_{0}>y_{d}$ is being assumed). Let us first analyze $\dot{y}_{1}(1)$. Since $h_{0}>y_{d}$, we have: $y(1)=y^{*}(1)=y_{d} \Rightarrow$ $v(0)=0 \Rightarrow L(0)=b+(M-b) \varphi(0) / \dot{y}_{d}$. Now, notice that $M>(m-e) /(1+e)$ for any $m \in[0, M)$ and $e \in[0,1]$. Then, since $a(0) \geqslant L(0)=b+(M-b) \varphi(0) / \dot{y}_{d}$, we have $a(0)>b+((m-e) /(1+e)-b) \varphi(0) / \dot{y}_{d} \Rightarrow \sigma \dot{y}_{d} a(0)+\alpha \varphi(0)$ $-\sigma b \dot{y}_{d}>0$, proving that $\dot{y}_{1}(1)>0$ (see (22)). Then $h_{1}>y_{d}$, giving rise to $y(2)=y^{*}(2)=y_{d}$. At this point, the second order dynamics of $\varepsilon_{1}(k)$ defined by (24), i.e.

$\varepsilon_{1}(k+2)=-p_{1} \varepsilon_{1}(k+1)-p_{0} \varepsilon_{1}(k)$

is valid (for $k=0$ ). Observe that since $y(1)=y^{*}(1)=y_{d}$, then $\varphi(1)=\dot{y}_{1}(1)$, and since $\varphi(1)=\dot{y}_{1}(1)>0$ and $\varphi(0)>0$, then $\varepsilon_{1}(1)>-\dot{y}_{d}$ and $\varepsilon_{1}(0)>-\dot{y}_{d}$. On the other hand, one can easily verify that for any $m \in[0, M)$, $e \in[0,1], b \in(-1,-0.5)$, and $c \in(b+0.5,0)$, we have: $0<-p_{1}<-p_{1}-p_{0}<1$. Hence, one sees that since $\varepsilon_{1}(1)>-\dot{y}_{d}$ and $\varepsilon_{1}(0)>-\dot{y}_{d}$, then: $\varepsilon_{1}(2)=$ $-p_{1} \varepsilon_{1}(1)-p_{0} \varepsilon_{1}(0)>\left(-p_{1}-p_{0}\right)\left(-\dot{y}_{d}\right)>-\dot{y}_{d}$. Hence $\dot{y}_{1}(2)>0$, and since $y(2)=y^{*}(2)=y_{d}$, then $h_{2}>y_{d}$ giving rise to $y(3)=y^{*}(3)=y_{d}$ which implies $\varphi(2)=\dot{y}_{1}(2)$. Then (26) is again valid (for $k=1$ ), and since $\varepsilon_{1}(2)>-\dot{y}_{d}$ and $\varepsilon_{1}(1)>-\dot{y}_{d}$, then: $\varepsilon_{1}(3)=-p_{1} \varepsilon_{1}(2)$ $-p_{0} \varepsilon_{1}(1)>\left(-p_{1}-p_{0}\right)\left(-\dot{y}_{d}\right)>-\dot{y}_{d}$, that is $\dot{y}_{1}(3)>0$. The whole process is then repeated at every impact, proving that $\dot{y}_{1}(k+1)>0, \forall k \geqslant 0$. Therefore $h_{k}>y_{d}, \forall k \geqslant 1$, which completes the proof (for the present case).

(2) Initial conditions such that $h_{0} \leqslant y_{d}$ : From the preceding analysis, one can easily realize that in this situation, by just ensuring $h_{1}>y_{d}$ and subsequently $h_{2}>y_{d}$, the asymptotically stable second order dynamics of $\varepsilon_{1}(k)$ in (26) is retrieved $\forall k \geqslant 1$, and consequently: $\lim _{k \rightarrow \infty} \varepsilon(k)=0$, and $y(k)=y^{*}(k)=y_{d}, \forall k \geqslant 2$. Then, all we need is to show that this is accomplished through the
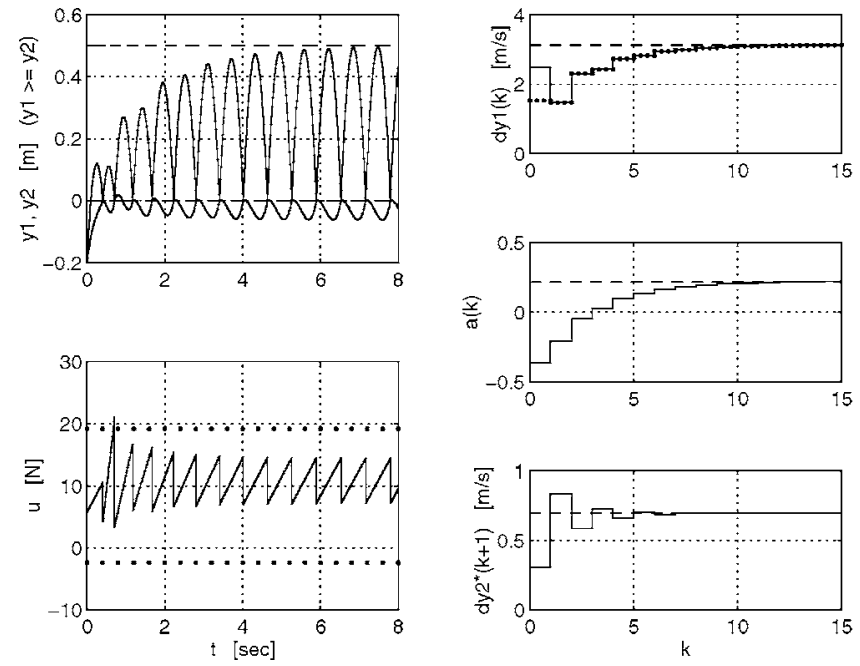

Fig. 3. Adaptive control: $h_{0}>y_{d}$.

choices of $a(0)$ and $a(1)$. Recalling that $M>(m-e) /$ $(1+e)$ for any $m \in[0, M)$ and $e \in[0,1]$, and since $a(0) \geqslant$ $L(0)=b+(1+M) v(0)+(M-b) \varphi(0) / \dot{y}_{d}$, we have:

$$
\begin{aligned}
& a(0)>b+\frac{\left(1+\frac{(m-e)}{(1+e)}\right) v(0)+\left(\frac{(m-e)}{(1+e)}-b\right) \varphi(0)}{\dot{y}_{d}} \\
& \Rightarrow \sigma \dot{y}_{d} a(0)+\alpha \varphi(0)-\sigma b \dot{y}_{d}>v(0) .
\end{aligned}
$$

Hence $\dot{y}_{1}(1)>v(0)($ see $(22)) \Rightarrow y(1)+\dot{y}_{1}^{2}(1) / 2 g>y_{d}$ (recall the definition of $v(k)$ and property 3$)$. Therefore $h_{1}>y_{d}$ (see (14)), giving rise to $y(2)=y^{*}(2)=y_{d} \Rightarrow$ $v(1)=0 \Rightarrow L(1)=b+(M-b) \varphi(1) / \dot{y}_{d}$. Finally, since $a(1) \geqslant L(1)=b+(M-b) \varphi(1) / \dot{y}_{d}, \quad$ then $a(1)>b+$ $((m-e) /(1+e)-b) \varphi(1) / \dot{y}_{d} \Rightarrow \sigma \dot{y}_{d} a(1)+\alpha \varphi(1)-\sigma b \dot{y}_{d}>$ 0 . Hence $\dot{y}_{1}(1)>0$ (see (22)), and since $y(2)=y^{*}(2)=y_{d}$, then $h_{2}>y_{d}$.

The following numerical examples show the results obtained from the application of the proposed adaptive scheme to a 1-dof juggler with the following parameter values: $e=0.8, m_{1}=0.1 \mathrm{~kg}$, and $m_{2}=1 \mathrm{~kg}$, giving $m=0.1$. The desired fixed-point is $\left(y_{d}, \dot{y}_{d}\right)=(0,3.13 \mathrm{~m} / \mathrm{s})$, resulting in a fixed apex $h_{d}=y_{d}+\dot{y}_{d}^{2} / 2 g=0.5 \mathrm{~m}$. The control parameter values were taken as $b=-0.9$, $c=-0.3$, and $M=0.2$. Figs. 3 and 4 show the results of the simulations for the two initial condition cases: $h_{0}>y_{d}$ and $h_{0} \leqslant y_{d}$, respectively. In the left-hand side of the figures, the position trajectories and the control input $u$ are presented. In the system trajectory curves, the lower and upper dashed lines indicate respectively the values of $y_{d}$ and $h_{d}$, while that in the input force graphs indicates the robot weight $\left(m_{2} g\right)$. The meaning of the dotted lines appearing in the force curves will be explained later. In the right-hand side of the figures, the discrete evolution of $\dot{y}_{1}(k), a(k)$, and $\dot{y}_{2}^{*}(k+1)$, are shown. The dashed lines indicate the desired convergence values: $\dot{y}_{d}, a^{*}$, and $a^{*} \dot{y}_{d}$, 

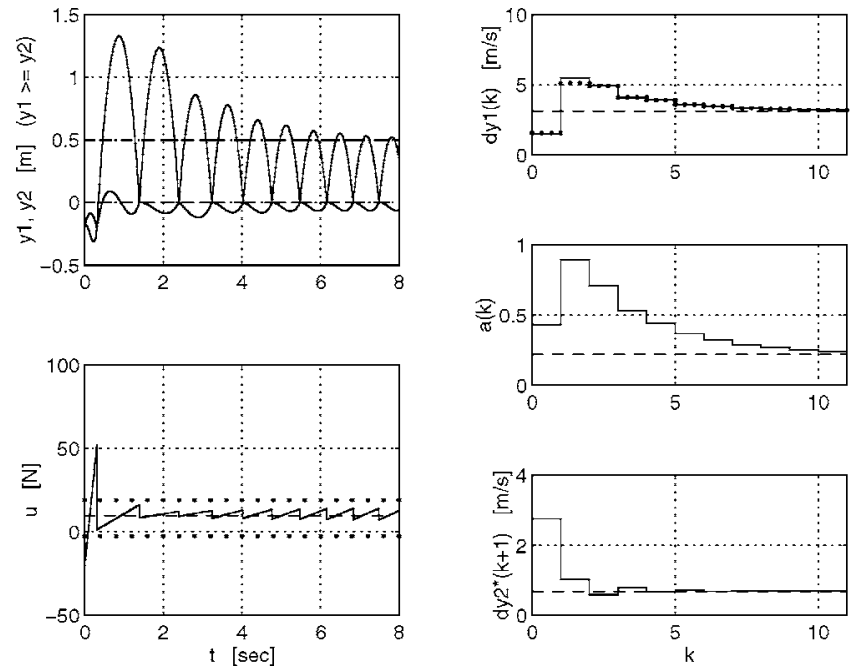

Fig. 4. Adaptive control: $h_{0} \leqslant y_{d}$.

respectively. In the $\dot{y}_{1}(k)$ graph, the dotted-line curve represents the evolution of $\varphi(k)$; observe that $\varphi(k)=y_{1}(k)>0, \forall k \geqslant i$, where $i=1$ if $h_{0}>y_{d}$, and $i=2$ if $h_{0} \leqslant y_{d}$. In the first example, Fig. 3, the following initial impact conditions were taken: $y(0)=-0.2 \mathrm{~m}, \dot{y}_{1}(0)=$ $2.5 \mathrm{~m} / \mathrm{s}$, and $\dot{y}_{2}(0)=1 \mathrm{~m} / \mathrm{s}$, giving $h_{0}=0.12 \mathrm{~m}>y_{d}$. The auxiliary state initial value was $a(0)=-0.36=L(0)$. In the second example, Fig. 4, everything was repeated except that this time the object initial impact velocity was taken as: $\dot{y}_{1}(0)=1.5 \mathrm{~m} / \mathrm{s}$, yielding $h_{0}=-0.1 \mathrm{~m}<y_{d}$. The auxiliary state initial values were selected as: $a(0)=0.43=L(0)$ and $a(1)=0.9=L(1)$. Observe that in both examples, $\dot{y}_{1}(k)$ and $a(k)$ converge asymptotically towards $\dot{y}_{d}$ and $a^{*}$ respectively, and $y(k)=y_{d}, \forall k \geqslant 2$ $\left(\forall k \geqslant 1\right.$ if $\left.h_{0}>y_{d}\right)$. Finally, let us point out an important observation concerning the upper and lower dotted lines in the force curves. These stand respectively for $U_{2}=m_{2} g+m_{2} V_{2}$ and $U_{1}=m_{2} g+m_{2} V_{1}$, where $V_{1} \triangleq[(-3+e-2 m+2 e m) /(1+e)] g \quad$ and $\quad V_{2} \triangleq$ $[(3-2 e+4 m-e m) /(1+e)] g$. In (Zavala-Río \& Brogliato, 1999), a control strategy ensuring the convergence of the object impact states to the desired fixed point avoiding saturation of the input $u$ when this one is bounded such that $u_{1} \leqslant u \leqslant u_{2}$ was proposed, based on the assumption that $u_{1}$ and $u_{2}$ are such that $u_{1}<U_{1}$ and $u_{2}>U_{2}$. The results observed in the present examples, Figs. 3 and 4, show that the lowest and highest values of $u$ at each flight time, $u\left(t_{k}^{+}\right)$and $u\left(t_{k+1}^{-}\right)$, are respectively higher than $U_{1}$ and lower than $U_{2}$ for all $k \geqslant 2$, i.e. $U_{1}<u\left(t_{k}^{+}\right)<u\left(t_{k+1}^{-}\right)<U_{2}, \forall k \geqslant 2$. This suggests that conditions on the initial system impact state values and on $a(0)$ can be found in order for the adaptive strategy in Proposition 2 to be applicable avoiding saturation of the input $u$ when this one is bounded such that $u_{1} \leqslant u \leqslant u_{2}$ where $u_{1}<U_{1}$ and $u_{2}>U_{2}$.

\section{Conclusions}

This note is devoted to the control of a class of nonsmooth mechanical systems, that encompasses simple models of juggling and hopping robots. Its aim is to extend some previously studied control algorithms (Zavala-Río \& Brogliato, 1999; Brogliato \& Zavala-Río, 2000). Roughly speaking, such controllers are constructed by first designing a sequence of fictitious inputs in terms of the "robot" pre-impact velocities (which constitutes a step that has attracted the interest of some researchers in the field (Buehler, Koditshek, \& Kindklmann, 1994)). Then in a second stage, one designs the force control input that guarantees that this pre-impact velocities sequence is realized. The note focuses mainly on the first step. It concerns the relaxation of the a priori knowledge of physical parameters like the kinematic restitution coefficient $e$ and the object's mass, in the design of the robot pre-impact velocities. A semi-globally stable adaptive scheme is proposed. Some numerical simulations illustrate the theoretical results.

\section{References}

Babitsky, V. I. (1998). Theory of vibro-impact systems and applications. Foundations on engineering mechanics. Berlin: Springer.

Bhattacharyya, S. P., Chapellat, H., \& Keel, L. H. (1995). Robust control. The parametric approach. Englewood Cliffs, NJ: Prentice-Hall.

Brach, R. M. (1991). Mechanical impact dynamics. Rigid body collisions. New York: Wiley Interscience Publications and Wiley.

Brogliato, B. (1999). Nonsmooth mechanics. Models, dynamics and control. CCES (2nd ed.). London: Springer. Erratum and addenda available at http://www-lag.ensieg.inpg.fr/publications.html.

Brogliato, B., \& Zavala-Río, A. (2000). On the control of complementary-slackness juggling mechanical systems. IEEE Transactions on Automatic Control, 45, 235-246.

Buehler, M., Koditshek, D. E., \& Kindklmann, P. J. (1994). Planning and control of robotic juggling and catching tasks. International Journal of Robotics Research, 13, 101-118.

ten Dam, A. A., Dwarshuis, E., \& Willems, J. C. (1997). The contact problem for linear continuous-time dynamical systems: a geometric approach. IEEE Transactions on Automatic Control, 42, 458-472.

Masri, S. F., \& Caughey, T. K. (1966). On the stability of the impact damper. ASME Journal of Applied Mechanics, 33, 586-592.

Rockafellar, R. T. (1970). Convex analysis. Princeton landmarks in mathematics. Princeton, NJ: Princeton University Press (10th printing 1997).

van der Schaft, A., \& Schumacher, J. M. (1996). The complementarity-slackness class of hybrid systems. Mathematics of Control, Signal and Systems, 9, 266-301.

Shaw, S. W., \& Rand, R. H. (1989). The transition to chaos in a simple mechanical system. International Journal of Non-linear Mechanics, 24, 41-56.

Wang, Y. (1993). Dynamic modeling and stability analysis of mechanical systems with time-varying topologies. ASME Journal of Mechanics Design, 115, 808-816.

Zavala-Río, A. (1997). Commande de robots jongleurs. Ph.D. thesis, Laboratoire d'Automatique de Grenoble, ENSIEG, INPG, France.

Zavala-Río, A., \& Brogliato, B. (1999). On the control of a one-degreeof-freedom juggling robot. Dynamics and Control, 9, 67-90. 